\title{
Impact of congenital color vision defect on color-related tasks among schoolchildren in Durban, South Africa
}

This article was published in the following Dove Press journal:

Clinical Optometry

\section{Khathutshelo Percy Mashige (D)}

University of KwaZulu-Natal, School of Health Science, Discipline of Optometry, Durban 4000, South Africa
Correspondence: Khathutshelo Percy Mashige

University of KwaZulu-Natal, School of Health Science, Discipline of Optometry, Private Bag X5400I, Durban 4000, South Africa

Tel +273I 2607986

Email mashigek@ukzn.ac.za
Background: People with congenital color vision deficiency can experience some difficulties with seeing colors in everyday life, and these problems vary according to the nature and severity of the defect, the individual's circumstances and their ability to develop coping or adaptive strategies.

Purpose: To determine the impact of congenital color vision deficiency on color-related tasks among Black South African schoolchildren.

Methods: A cross-sectional descriptive study was conducted among 1305 public schoolchildren in Durban, South Africa. Structured questionnaires were administered to determine the difficulties they encountered in aspects of color matching and recognition.

Results: Of the 1305 schoolchildren who participated, 601 (46.1\%) were boys and 704 $(53.9 \%)$ were girls, with $29(2.2 \%)$ having congenital color vision defects. The prevalence of congenital color vision deficiency was higher in boys $(n=25 ; 4.2 \%)$ than in girls $(n=4 ; 0.6 \%)$. A statistically significant proportion $(P<0.0001)$ reported difficulties with color-related schoolwork and activities of daily living.

Conclusion: Congenital color vision deficiency affects many aspects of life and extends across play, sports, education, health and safety issues. Early detection of children with congenital color vision defect through regular school screenings is needed to offer affected children appropriate vocation and career guidance.

Keywords: color vision, color vision defect, congenital, color-related tasks, South Africa

\section{Introduction}

Color vision is an integral part of a child's life as they are often exposed to colorful objects at school and during day-to-day activities. ${ }^{1}$ For example, they are asked to describe certain items by their colors and fill in coloring sheets at school. ${ }^{2,3}$ Children with CCVD are not able to learn to their full capacity, which can undermine their confidence and provide a faulty foundation for future learning. ${ }^{2,3}$ Children with CCVD, except for a few mildly affected deuteranomals, have problems with color-related tasks in this increasingly colorful world. ${ }^{2}$

Although more likely to guess more colors, a child with deuteranomaly (the most common form of CCVD) will only be able to accurately name four colors within a box of 24 colored pencils. ${ }^{4}$ As a result, parents of children with CCVD have often reported that their children were slow learners and were consequently ridiculed for confusing colors and coloring objects wrongly. ${ }^{5,6}$ As pupils progress through school, there is an increasing use of colors, such as interpreting colored 
maps and graphs; colors used to highlight material; in the science laboratory, art room, mathematics, food technology, information technology and even history. ${ }^{3}$

Activities such as interpersonal interactions are important for a child's social development, with CCVD children facing difficulties when they cannot differentiate between teams based on team colors. ${ }^{6}$ For example, in the sporting arena, a player with CCVD might pass a ball to the opposition due to color confusion with the vest. Similarly, balls are often colored to create contrast with the grass, but may not be visible to those with CCVD. While these students can learn to identify colors through their hue and saturation, they still cannot actually see them. ${ }^{3}$ Steward and Cole ${ }^{7}$ reported CCVD-related difficulties during schooling years in a variety of sporting activities involving 102 affected players. These players were reported to lose orange golf balls in the grass, mistook red for brown snooker balls and failed to differentiate their team members from the opponents wearing colored uniforms. $^{4,7}$

CCVD is a common condition, which can also significantly impact the quality of life for health, lifestyle, emotions and occupations in adulthood. ${ }^{8}$ For instance, patients with CCVD reported difficulties with activities of daily living that involved color in their middle to late adulthood. ${ }^{9}$ These activities included difficulties with clothing colors, workplace/hobbies colors, natural colors, colors relating to cooking and sports colors. ${ }^{9}$ Similarly, CCVD patients in Steward and Cole $^{7}$ reported difficulties in everyday tasks such as workplace/hobbies colors, plant/ flower identification, ripeness of fruits and vegetables, and taking wrong medication due to color difficulties.

The prevalence of CCVD varies has been reported to vary from one locality to another for a variety of reasons such as racial, ethnic and geographical differences. ${ }^{10}$ CCVD has been reported to be $8 \%$ and $0.4 \%$ in European Caucasian males and female populations, respectively. ${ }^{10}$ Findings of previous reports vary from overall prevalence of $1.5 \%$ in Nigeria, ${ }^{11} 2.2 \%$ in $\operatorname{Iran}^{12}$ to $4.8 \%$ in Italy. $^{13}$

To the best of the author's knowledge, no studies have reported on the effects of CCVD on color-related tasks in children in South Africa. Hence, this study sets out to determine the difficulties encountered by schoolchildren with congenital color vision defect in school and daily activities. This would assist children with the defects to identify the domains of difficulties in school and daily living and to determine possible strategies to cope with their defect (as they are untreatable).

\section{Methods}

A descriptive cross-sectional study was carried out among schoolchildren in Durban, KwaZulu-Natal Province, South Africa. The study was conducted from April to September 2017. Schoolchildren were selected using a randomized, stratified, cluster sampling process from six districts of Durban. For logistical and operational reasons, sampling was restricted to districts within $1 \mathrm{hr}$ 's drive from the University of KwaZulu-Natal's Eye Clinic (where clinical examinations were performed). The sample size for the study was calculated by using the formula for estimating a single population proportion, with prevalence being set at $8 \%$ at a confidence interval of $95 \%$ and a $0.5 \%$ precision. $^{13}$ After adjusting for a nonresponse rate of $10 \%$, a minimum sample size of 1105 students was calculated. Exclusion criteria included compensated visual acuity of worse than $6 / 6$ in both eyes or color vision deficiency in one eye, which is not typical of congenital color deficiency. Children with ocular pathology, trauma, previous ocular surgery, longterm use of medication and those born to parents who are not from South Africa were also excluded from the study.

The assessment consisted of data regarding sociodemographic details, visual acuity, retinoscopy, subjective refraction, pen torch examination, direct ophthalmoscope observation and color vision testing. Visual acuity assessment was performed with a Tumbling E Snellen's chart in the optometry clinic, and all those with spectacles had their visual acuities assessed while wearing them. Retinoscopy and subjective refraction were done to determine refractive error, while pen torch and direct ophthalmoscopy through undilated pupils were performed for ocular health examination. The pseudo-isochromatic color plate test "Colour Vision Testing Made Easy" (CVTME) was used to assess color vision of all the children in an optometry clinic with standard illumination. The CVTME color plates were held $75 \mathrm{~cm}$ from the children and tilted so that the plane of the page was at right angles to the participant's line of vision. ${ }^{14}$ Each child was asked to read the numbers on the first 14 plates of the test booklet, at $4 \mathrm{~s}$ per plate. All the testing was conducted under binocular viewing conditions, and the test was performed twice for all children. A child who made more than three errors between plates 1 and 14 during the first and/or second test sessions was judged to have failed the screening and retested a third time. Each 
child was considered having CCVD if they had more than three errors at two out of three sessions. The questionnaire used in this study was adapted from the study by Ugalahi et al. ${ }^{15}$ A pilot study was conducted among 20 schoolchildren outside the study area who did not take part in the final study in order to critically evaluate and finalize the questionnaire. Thereafter, structured questionnaires were administered to all children to determine the difficulties they encountered in aspects of color-related school tasks and other daily activities.

Ethical approval to conduct the study was obtained from the Biomedical Research Ethics Committee of the University of KwaZulu-Natal. Permission to conduct the study was also obtained from community leaders and the school principals in the selected areas. Parents and/or legal guardians of the schoolchildren who participated in this study signed consent forms, while the children provided informed assent. Those found to have CCVD were advised about their condition and how it may affect their future choice of occupation or profession, as well as any other conditions that were identified during the various tests. In addition, the parents/legal guardians of children who were found to have CCVD were given feedback about their children's color vision status.

Statistical analysis was conducted using the Statistical Package for Social Sciences program (SPSS for Windows, version 24; SPSS Inc., Chicago, Illinois, USA). Descriptive statistics was used to calculate means, frequencies and proportions. Fisher's exact test was used to determine associations between variables as the frequencies in a given category were small.

\section{Results}

All the children selected agreed to participate in the study, which gave a response rate of $100 \%$. Thus, data was analyzed for 1305 children, including 704 (53.9\%) girls and $601(46.1 \%)$ boys, with a mean age of 12.06 \pm 1.8 years. Twenty nine $(2.2 \%)$ schoolchildren had CCVD and 1276 (97.8\%) had normal color vision (NCV). The prevalence of CCVD was higher in boys $25(4.2 \%)$ than girls $4(0.6 \%)$. The prevalence of CCVD was higher in the 13-17 year-olds (2.7\%) compared to the 7-12 year-olds $(1.7 \%)$. However, this difference was statistically insignificant $(P=0.12)$. Nineteen $(1.5 \%)$ of the 1305 schoolchildren expressed difficulty working on the computer and 15 (1.1\%) had difficulty identifying colors in crafts and hobbies, while $13(0.9 \%)$ had difficulties with selecting colors of clothes (Table 1).

A cross tabulation of CCVD children with NCV children showed that there was a statistically significant number of color deficient children who had difficulties with color-related activities both at school and in their daily living tasks $(P<0.001)$ (Table 2$)$.

\section{Discussion}

It has been reported that CCVD affects many aspects of life from childhood to adulthood, including play, sports, driving, education, occupation, and health and safety issues. ${ }^{1}$ CCVD is therefore a disability, with many people not being aware of their status until they face difficulties differentiating colors. ${ }^{3}$ This report present findings of the impact of CCVD on color-related activities among public schoolchildren in Durban, South Africa. The results of

Table I Difficulties with color-related tasks reported by schoolchildren

\begin{tabular}{|c|c|c|c|c|}
\hline Variables & $\begin{array}{l}\text { Never } \mathbf{N} \\
(\%)\end{array}$ & $\begin{array}{l}\text { Occasionally N } \\
\text { (\%) }\end{array}$ & $\begin{array}{l}\text { All the time } \mathrm{N} \\
\text { (\%) }\end{array}$ & $\begin{array}{l}\text { Total N } \\
\text { (\%) }\end{array}$ \\
\hline Colors and picture charts in mathematics text & $1298(99.5)$ & $5(0.4)$ & $2(0.1)$ & $1305(100)$ \\
\hline Working on the computer & $1286(98.6)$ & II (0.8) & $8(0.6)$ & $1305(100)$ \\
\hline Colors in fine arts & $1294(99.2)$ & $7(0.5)$ & $4(0.3)$ & $1305(100)$ \\
\hline Colors in crafts and hobbies & $1290(98.9)$ & $8(0.6)$ & $7(0.5)$ & $1305(100)$ \\
\hline $\begin{array}{l}\text { Identifying houses or teams during inter-house sports based on color of } \\
\text { jersey }\end{array}$ & $1299(99.6)$ & $4(0.3)$ & $2(0.1)$ & $1305(100)$ \\
\hline Selecting colors of clothes & $1292(99)$ & $9(0.7)$ & $4(0.3)$ & $1305(100)$ \\
\hline Identifying flowers based on colors & $1297(99.4)$ & $3(0.2)$ & $5(0.4)$ & $1305(100)$ \\
\hline Judging ripeness of fruit and vegetables based on colors & $1299(99.6)$ & $2(0.1)$ & $4(0.3)$ & $1305(100)$ \\
\hline Watching sports because of color of team jersey & $1294(99.2)$ & $4(0.3)$ & $7(0.5)$ & $1305(100)$ \\
\hline Recognizing and describing cars based on colors & $1296(99.4)$ & $7(0.5)$ & $2(0.1)$ & $1305(100)$ \\
\hline Recognizing traffic signal lights & $1298(99.5)$ & $3(0.2)$ & $4(0.3)$ & $1305(100)$ \\
\hline
\end{tabular}


Table 2 Difficulties with color-related tasks reported by 29 CCVD and I 276 NCV children

\begin{tabular}{|c|c|c|c|c|c|}
\hline \multirow[t]{2}{*}{ Variables } & \multicolumn{3}{|c|}{ Color-deficient (\%) } & \multirow[t]{2}{*}{ Fisher's exact test } & \multirow[t]{2}{*}{ Significance ( $P$-value) } \\
\hline & Yes & No & Total & & \\
\hline \multicolumn{6}{|c|}{ Identifying colors and charts in mathematics } \\
\hline Some difficulty & $5(7 \mid .4)$ & $2(28.6)$ & $7(100)$ & 236.4 & $<0.0001$ \\
\hline No difficulty & $15(1.6)$ & $1283(98.8)$ & $1298(100)$ & & \\
\hline \multicolumn{6}{|c|}{ Working on the computer } \\
\hline Some difficulty & $12(63.2)$ & $7(36.8)$ & $19(100)$ & 270.7 & $<0.0001$ \\
\hline No difficulty & II (0.9) & $1275(99.1)$ & $1286(100)$ & & \\
\hline \multicolumn{6}{|l|}{ Colors in fine arts } \\
\hline Some difficulty & $8(72.7)$ & $3(27.3)$ & II $(100)$ & 164.9 & $<0.0001$ \\
\hline No difficulty & $13(1.0)$ & $128 \mid(99.0)$ & $1294(100)$ & & \\
\hline \multicolumn{6}{|c|}{ Identifying colors in crafts and hobbies } \\
\hline Some difficulty & $9(60)$ & $6(40)$ & $15(100)$ & 241.6 & $<0.0001$ \\
\hline No difficulty & $15(1.2)$ & $1275(98.8)$ & $1290(100)$ & & \\
\hline \multicolumn{6}{|c|}{ Identifying different houses or teams during interhouse sports based on color of jersey } \\
\hline Some difficulty & $4(66.7)$ & $2(33.3)$ & $6(100)$ & 138.6 & $<0.0001$ \\
\hline No difficulty & $10(0.8)$ & $1289(99.2)$ & $1299(100)$ & & \\
\hline \multicolumn{6}{|c|}{ Selecting colors of clothes } \\
\hline Some difficulty & $8(6 \mid .5)$ & $5(38.5)$ & $13(100)$ & 248.1 & $<0.0001$ \\
\hline No difficulty & $14(1.1)$ & $1278(98.9)$ & $1292(100)$ & & \\
\hline \multicolumn{6}{|c|}{ Identifying flowers based on colors } \\
\hline Some difficulty & $5(62.5)$ & $3(37.5)$ & $8(100)$ & 190.5 & $<0.0001$ \\
\hline No difficulty & $13(1.0)$ & $1284(99.0)$ & $1297(100)$ & & \\
\hline \multicolumn{6}{|c|}{ Judging ripeness of fruits and vegetables } \\
\hline Some difficulty & $4(66.7)$ & $2(33.3)$ & $6(100)$ & 144.4 & $<0.0001$ \\
\hline No difficulty & $16(1.2)$ & $1283(98.8)$ & $1299(100)$ & & \\
\hline \multicolumn{6}{|c|}{ Watching sports because of colors } \\
\hline Some difficulty & $9(81.8)$ & $2(18.2)$ & II $(100)$ & 252.1 & $<0.0001$ \\
\hline No difficulty & II (0.9) & $1283(99.1)$ & $1294(100)$ & & \\
\hline \multicolumn{6}{|c|}{ Describing and recognizing cars based on colors } \\
\hline Some difficulty & $6(66.7)$ & $3(33.3)$ & $9(100)$ & 188.2 & $<0.000$ I \\
\hline No difficulty & $15(1.2)$ & I28I (98.8) & $1296(100)$ & & \\
\hline \multicolumn{6}{|c|}{ Recognizing traffic signal light } \\
\hline Some difficulty & $5(7 \mid .4)$ & $2(28.6)$ & $7(100)$ & 233.8 & $<0.0001$ \\
\hline No difficulty & $18(1.4)$ & $1280(98.6)$ & $1298(100)$ & & \\
\hline
\end{tabular}

this study found that although the prevalence of CCVD was relatively low $(2.2 \%)$, the impact on color-related activities was significant. A significant number of CCVD children reported more difficulties with color- related school and activities of daily living tasks compared to normal color vision children.

The prevalence of CCVD was $2.2 \%$, which is similar to findings reported in other studies conducted in 
Africa. ${ }^{15,16}$ Although the prevalence has been reported to be increasing among Africans, it is still lower than those of Asians and Caucasians. ${ }^{10}$

Children with CCVD in this study encountered more difficulties performing color-related tasks than their normal color vision counterparts. This can be an impediment in certain subjects, especially the sciences. ${ }^{1}$ Sullivan $^{4}$ reported that children with CCVD tend to lag behind in mathematics, science, geography, reading, sport and food technology, as they are unable to appreciate how colors are used in art and use the wrong colors when painting. ${ }^{4}$ For instance, in chemistry, CCVD children are unable to read litmus paper accurately or identify the colors of different chemical solutions in quantitative analysis. In biology, they are unable to accurately read stained slides under a microscope, and may not be able to identify species of plants and carry out dissections. In physics, they have difficulty with colored wiring and use of prisms, and are unable to read pie charts and graphs in mathematics. $^{3,5,6,17}$

A statistically significant proportion $(P<0.0001)$ of children with CCVD had difficulty with computers, crafts and hobbies, suggesting that good color identification is required to perform these color-related tasks. Campbell et $\mathrm{al}^{18}$ reported that there were statistically significant differences between the CCVD medical practitioners and those with normal color vision (NCV) in their ability to outline abnormalities in five of the six photographs that showed fresh blood, a photograph of a rash, and marking the position of bacilli in the photograph of a stained slide. In the area of sports, a statistically significant proportion of children with CCVD reported having more difficulty with recognizing the colors of jerseys during inter-house sporting competitions than NCV children $(P<0.0001)$. Harris and Cole ${ }^{19}$ concluded that

\begin{abstract}
Abnormal colour vision is a modest handicap to playing cricket and that cricketers with abnormal colour vision, especially those with a severe or a protan deficiency, tend not to reach the highest levels of cricket. They will have most difficulty with fielding.
\end{abstract}

More children with CCVD reported difficulties in the area of color-related activities of daily living compared to those with NCV $(P<0.0001)$, these findings being similar to previous reports. ${ }^{7,9,15,20}$ For example, Ugalahi et $\mathrm{al}^{15}$ found that CCVD students had significant difficulty with selecting colors of clothes, identifying flowers, judging the ripeness of fruits and vegetables, and recognizing and describing cars based on colors, watching sports, and identifying traffic signal lights compared with NCV students $(P<0.0001)$. CCVD children may therefore face difficulties when playing with others due to their inability to differentiate between different teams by colored tags, or colored pieces in board games. ${ }^{6}$ CCVD may also affect the personal safety of children as they may get lost if directions are given using colored objects as signs. ${ }^{6}$ It may also affect the nutritional status of children, as they may be "fussy" about eating certain foods (especially fruits and vegetables) because they perceive these foods to have unpalatable colors. ${ }^{4}$ The difficulties encountered by schoolchildren in recognizing traffic signal lights have also been reported in other studies, ${ }^{21}$ which have shown that protans have reduced ability to recognize red signals and deuteranopes to recognize red, orange and green colorcoded traffic control devices.

\section{Conclusion}

CCVD children encounter various difficulties with colorrelated schoolwork and activities of daily living. This disability not only has implications for their childhood, but for the adult life and career choices, and needs to be identified and managed appropriately. More research therefore needs to be done to identify CCVD and its potential implications in order to implement measures to overcome these difficulties during the early school years.

\section{Acknowledgments}

The author is a University of KwaZulu-Natal (UKZN) Developing Research Innovation, Localisation and Leadership in South Africa (DRILL) fellow. DRILL is a NIH D43 grant (D43TW010131) awarded to UKZN in 2015 to support a research training and induction program for early career academics. The content is solely the responsibility of the author and does not necessarily represent the official views of DRILL and the National Institutes of Health. The author thanks all the participants who took part in this study.

\section{Disclosure}

The author reports no conflicts of interest in this work.

\section{References}

1. Chan XBV, Goh SMS, Tan NC. Subjects with colour vision deficiency in the community: what do primary care physicians need to know? Asia Pac Fam Med. 2014;13:10. doi:10.1186/s12930-0140010-3 
2. Cole BL. The handicap of abnormal colour vision. Clin Exp Optom. 2004;87(4-5):258-275. doi:10.1111/j.1444-0938.2004.tb05056.x

3. Albany-Ward K. Why colour really does matter. In: Prep School. Vol. 15. 2011:36-37. Available from: http:www.colourblindawareness. org/.../Prep-School-SEN-Summer-201.

4. Sullivan K. Colour-blind children. Spec Educ Needs. 2011;12:21-23.

5. About color blindness (Color vision deficiency): life's minor frustrations (and occasional dangers) for the colour blind. Available from: http://www.toledo-bend.com/colorblind/aboutCB. asp. Accessed July 30, 2019.

6. Testing color vision: frequently asked questions. Available from: http://colorvisiontesting.com/color7.htm. Accessed July 30, 2019.

7. Steward JM, Cole BL. What do colour vision defectives say about everyday tasks?. Optom Vis Sci. 1989;66(5):288-295. doi:10.1097/ 00006324-198905000-00006

8. Barry JA, Mollan S, Burdon MA, Jenkins M, Denniston AK. Development and validation of a questionnaire assessing the quality of life impact of Colour Blindness (CBQoL). BMC Ophthalmol. 2017;17:179. doi:10.1186/s12886-017-0579-z

9. Tagarelli A, Piro A, Tagarelli G, Lantieri PB, Risso D, Olivieri RL. Colour blindness in everyday life and car driving. Acta Ophthalmol Scand. 2004;82(4):436-442. doi:10.1111/aos.2004.82.issue-4

10. Birch J. Worldwide prevalence of red-green colour deficiency. J Opt Soc Am A Opt Image Sci Vis. 2012;29(3):313-320. doi:10.1364/ JOSAA.29.000313

11. Abah ER, Oladigbolu KK, Samaila E, Gani Ikilama A. Ocular disorders in children in Zaria children's school. Niger J Clin Pract. 2011;14:473-476. doi:10.4103/1119-3077.91759

12. Rajavi Z, Sabbaghi H, Baghini AS, Yaseri M, Sheibani K, Norouzi G. Prevalence of colour vision deficiency and its correlation with amblyopia and refractive errors among primary school children. $J$ Ophthalmic Vis Res. 2015;10:130-138. doi:10.4103/2008322X.176909
13. Gallo PG, Panzo M, Lantieri PB, et al. Some psychological aspects of colour blindness at school: a field study in Calabria and Basilicata (southern Italy). Colour Res Appl. 2003;28:216-220. doi:10.1002/col.10148

14. Cotter SA, Lee DY, French AL. Evaluation of a new colour vision test: "colour vision testing made easy". Optom Vis Sci. 1999;76:631636. doi:10.1097/00006324-199909000-00020

15. Ugalahi MO, Fasina O, Ogun OA, Ajayi BG. Prevalence of congenital colour vision deficiency among secondary school students in Ibadan, South-West Nigeria. Niger Postgrad Med J. 2016;23(1):9396. doi:10.4103/1117-1936.186301

16. Rahman SA, Singh PN, Nanda PK. Comparison of the incidence of colour blindness between sections of Libyan and Indian populations. Indian J Physiol Pharmacol. 1998;42:271-5.

17. Spalding JAB. Doctors with inherited colour vision deficiency: their difficulties in clinical work. In: Cavonius CR, editor. Colour Vision Deficiencies XIII. Vol. 59. The Netherlands: Springer Netherlands; 1997:483-489.

18. Campbell JL, Griffin L, Spalding JA, Mir FA. The effect of abnormal colour vision on the ability to identify and outline coloured clinical signs and to count stained bacilli in sputum. Clin Exp Optom. 2005;88:3763-3781. doi:10.1111/j.1444-0938.2005.tb05103.x

19. Harris RW, Cole BL. Abnormal colour vision is a handicap to playing cricket but not an insurmountable one. Clin Exp Optom. 2007;90:4514-4556. doi:10.1111/j.1444-0938.2006.00180.x

20. Chia A, Gazzard G, Tong L, et al. Red-green colour blindness in Singaporean children. Clin Exp Ophthalmol. 2008;36(5):464-467.

21. Atchison DA, Pedersen CA, Dain SJ, Wood JM. Traffic signal colour recognition is a problem for both protan and deutan colourvision deficients. Hum Factors. 2003;45(3):495-503. doi:10.1518/ hfes.45.3.495.27247

22. Kirkwood BR, Sterne JA. Essentials of Medical Statistics. 2nd ed. Massachusetts: Blackwell Science Limited; 2003:420.

\section{Clinical Optometry}

\section{Publish your work in this journal}

Clinical Optometry is an international, peer-reviewed, open access journal publishing original research, basic science, clinical and epidemiological studies, reviews and evaluations on clinical optometry. All aspects of patient care are addressed within the journal as well as the practice of optometry including economic and business analyses. Basic and clinical research papers are published that cover

Submit your manuscript here: https://www.dovepress.com/clinical-optometry-journa all aspects of optics, refraction and its application to the theory and practice of optometry. The manuscript management system is completely online and includes a very quick and fair peer-review system, which is all easy to use. Visit http://www.dovepress.com/ testimonials.php to read real quotes from published authors. 